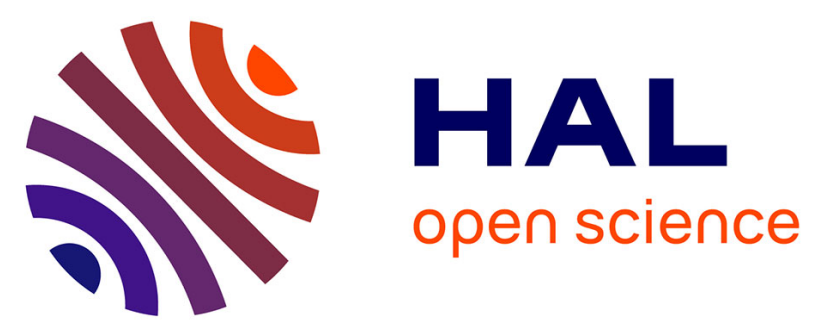

\title{
Self-propulsion of symmetric chemically active particles: Point-source model and experiments on camphor disks
}

Dolachai Boniface, Cécile Cottin-Bizonne, Ronan Kervil, Christophe Ybert, François Detcheverry

\section{To cite this version:}

Dolachai Boniface, Cécile Cottin-Bizonne, Ronan Kervil, Christophe Ybert, François Detcheverry. Self-propulsion of symmetric chemically active particles: Point-source model and experiments on camphor disks. Physical Review E , 2019, 99 (6), pp.062605. 10.1103/PhysRevE.99.062605 . hal02172145

\section{HAL Id: hal-02172145 \\ https://hal.science/hal-02172145}

Submitted on 3 Jul 2019

HAL is a multi-disciplinary open access archive for the deposit and dissemination of scientific research documents, whether they are published or not. The documents may come from teaching and research institutions in France or abroad, or from public or private research centers.
L'archive ouverte pluridisciplinaire HAL, est destinée au dépôt et à la diffusion de documents scientifiques de niveau recherche, publiés ou non, émanant des établissements d'enseignement et de recherche français ou étrangers, des laboratoires publics ou privés. 


\title{
Self-propulsion of symmetric chemically active particles: Point-source model and experiments on camphor disks
}

\author{
Dolachai Boniface, Cécile Cottin-Bizonne, Ronan Kervil, Christophe Ybert, and François Detcheverry \\ Univ Lyon, Université Claude Bernard Lyon 1, CNRS, Institut Lumière Matière, F-69622 Villeurbanne, France
}

(Received 12 February 2019; published 17 June 2019)

\begin{abstract}
Solid undeformable particles surrounded by a liquid medium or interface may propel themselves by altering their local environment. Such nonmechanical swimming is at work in autophoretic swimmers, whose selfgenerated field gradient induces a slip velocity on their surface, and in interfacial swimmers, which exploit unbalance in surface tension. In both classes of systems, swimmers with intrinsic asymmetry have received the most attention but self-propulsion is also possible for particles that are perfectly isotropic. The underlying symmetry-breaking instability has been established theoretically for autophoretic systems but has yet to be observed experimentally for solid particles. For interfacial swimmers, several experimental works point to such a mechanism, but its understanding has remained incomplete. The goal of this work is to fill this gap. Building on an earlier proposal, we first develop a point-source model that may be applied generically to interfacial or phoretic swimmers. Using this approximate but unifying picture, we show that they operate in very different regimes and obtain analytical predictions for the propulsion velocity and its dependence on swimmer size and asymmetry. Next, we present experiments on interfacial camphor disks showing that they indeed self-propel in an advection-dominated regime where intrinsic asymmetry is irrelevant and that the swimming velocity increases sublinearly with size. Finally, we discuss the merits and limitations of the point-source model in light of the experiments and point out its broader relevance.
\end{abstract}

DOI: 10.1103/PhysRevE.99.062605

\section{INTRODUCTION}

From the bacteria rotating their flagella to the blue whale whipping its tail, deforming the whole body or moving a specific appendage may be the most widespread swimming strategy in nature. All creatures that fly, walk, crawl, or slither also depend on mechanical actuation for their displacement [1]. Yet propulsion is possible without moving parts or change in body shape. Perhaps the simplest instance is the rocket, whose thrust is produced by strongly expelled exhaust gas. With all energy stored within the propeller, such means of propulsion is completely autonomous and operates even in a vacuum. The presence of a surrounding medium, such as liquid water, opens a new possibility for self-propulsion: altering the local environment to induce motion. Among the systems relying on this working principle, two important classes are the autophoretic and interfacial swimmers [2]. Since the mid-2000s, both have received sustained attention [3-7].

Autophoretic propulsion exploits the phenomena occurring at the liquid-solid interface when a swimmer generates in its vicinity an electric, concentration, or temperature field which is not uniform [8]. Fluid-solid interactions in the presence of a field gradient generates a flow within a thin layer near the solid resulting in an effective slip velocity on the particle. That such phoretic processes enable self-propulsion by nonmechanical means has been recognized at least since the 1960s, when an electrophoretic mechanism was suggested to rationalize the swimming of cyanobacteria devoid of flagellum $[9,10]$. However, it was not before the turn of this century that autophoretic motion was fully exploited to obtain artificial microswimmers $[11,12]$. The type of system that has attracted the most attention so far is probably the Janus particle, whose two hemispheres have distinct properties. For instance, they can differ in chemical activity: Whereas one face is inert, the other is Pt covered and catalyzes the decomposition of peroxide present in the solution, thus establishing a permanent concentration gradient. Janus particles whose hemispheres are maintained at distinct temperatures through different absorption of the illuminating light can give rise to thermophoresis [13], or when placed in a near-critical binary mixture, to local demixing and self-diffusiophoresis [14].

The class of interfacial self-propellers relies on the modification of forces present at the air-liquid interface. Through continuous release of chemical or heat, the surface tension around the swimmer is made nonuniform and the resulting unbalance in forces propels the particle forward. The history of the phenomena is a long one, with first reports of spontaneous motion of camphor grains at the surface of water dating back to 1756 [15]. By 1841, even though a dozen of authors had already investigated the topic, the mechanism of self-propulsion was still vigorously debated [16,17]. Camphor boats later made their way into the popular science literature $[18,19]$, and to this day, the concept has elicited numerous variations, from vapor-driven boats [20] to alcoholfilled edible "cocktail boats" [21]. In contrast to the phoretic mechanism, interfacial propulsion is unambiguously known to be exploited by some living organisms: The millimetric aquatic bug Microvelia can release an insoluble surfactant and reach a speed of $17 \mathrm{~cm} \mathrm{~s}^{-1}$, and some terrestrial insects use a similar strategy to return to land when accidentally fallen on the surface of water [22,23]. The motion, however, is only transient. Steady propulsion in artificial systems is generally made possible with a volatile surfactant that avoids saturation by quickly evaporating from the surface [7]. 
With the current interest in active matter, both autophoretic and interfacial swimmers have attracted attention as model systems to investigate collective and nonlinear effects, including clustering [24-26], oscillations, synchronization and pattern formation [5,27], density waves [28], swarming [29], and self-assembly [30]. Yet, in spite of a wealth of investigations $[4,5,7]$, some questions remain on the mechanism of individual self-propulsion. One aspect lacking a complete picture so far is the motion of isotropic swimmers permitted by spontaneous symmetry breaking. In this category of system, the particle "activity," be it chemical or heat release, is uniformly distributed and the asymmetry is induced by the motion itself through advection of the field.

The symmetry-breaking mechanism in autophoretic swimmers could not be captured in early theoretical descriptions that neglected advection entirely [31]. Intrinsic asymmetry of particles was therefore deemed necessary for self-propulsion. The effect of a finite Péclet number was subsequently investigated [32-34]. The possibility of symmetry breaking was put on firm theoretical ground by Michelin et al. [35] and also confirmed in molecular simulations [36]. However, to the best of our knowledge [37,38], there seem to be no experimental demonstration [39]. While swimming of isotropic droplets has been reported and explained [40-43], the propulsion in this case relies essentially on Marangoni flows at the droplet surface and requires internal flows within the swimmer. For isotropic solid particles, autophoretic motion is thus theoretically understood but not observed. For interfacial swimmers, the situation is somewhat reversed. There are several reports of isotropic swimmers such as camphor, benzoquinone, or phenanthroline disks [44-49]. Yet, despite much progress [6,50-52], the theoretical description of propulsion is not yet complete. Two basic questions have remained unanswered. (i) Can we predict the propulsion speed? Does it depend on swimmer size? (ii) Is the symmetry-breaking propulsion robust against unavoidable defects? Is it really operative in an experimental system?

In this work, we provide positive answers to these questions. We do so by combining simple modeling and experiments on camphor disks. First, building on earlier works [53], we develop to its full potential a point-source model. Though interfacial and phoretic swimmers rely on distinct mechanisms - the latter is force-free while the former is not-both can be treated within a common approach. We investigate the propulsion speed and discuss its dependence on the swimmer size, the dimension of the field, and the presence or absence of asymmetry. Confronting these predictions to experiments on interfacial symmetric swimmers-camphor disks-we confirm that they operate in a regime which is dominated by advection and where small asymmetries only play a minor role. Finally, we discuss the approximations of the point-source model and point out its relevance to address a variety of phenomena.

\section{POINT-SOURCE MODEL}

\section{A. Principle and notations}

To motivate the introduction of an approximate treatment, let us first underline the complexity of the problem under investigation, both for the phoretic and interfacial swimmers. In the former, the flow-through advection of the released chemical-affects the concentration and in turn the local slip velocity that serves as a boundary condition for the flow. Such a coupling between the hydrodynamic and the advectiondiffusion phenomena makes an analytical treatment difficult. One consequence is that in establishing the symmetrybreaking mechanism [35], only the threshold value can be expressed explicitly. Numerical approaches are necessary to obtain the swimming velocity. For the interfacial swimmer, a similar interdependence arises between the velocity field and the concentration around the particle, which sets the surface forces driving the motion. Besides, there is the additional complication that a nonuniform distribution of surfactant on the free surface induces Marangoni stresses affecting the flow underneath. Again, a complete analytical treatment would be a formidable task and some restrictions are unavoidable. For instance, the analytical investigation of an asymmetric interfacial swimmer [54] is limited to small Reynolds and Péclet numbers. The basic idea of the point-source model is to decouple the hydrodynamics and advection-diffusion problems by evaluating the concentration field in a very simple situation: a punctual swimmer that leaves the flow undisturbed and thus uniform. An alternate but equivalent formulation is that the concentration field is described by its far-field expression, i.e., retains only the lowest order in a multipole expansion. The phenomena induced by the presence of a solid impenetrable surface and the departure from a uniform flow in the vicinity of the swimmer are thus entirely discarded. Even though this may appear as a very crude simplification, it renders the problem tractable and leads to surprisingly meaningful predictions.

As far as we are aware, the point-source model was first introduced by Mikhailov and Meinköhn [53,55] to describe an interfacial swimmer which releases a chemical diffusing in the underlying bulk fluid. More recent works consider the one-dimensional situation $[5,7,56]$ relevant when swimmers are confined within a narrow channel. A similar approach was also used to interpret molecular simulations of a symmetric autophoretic swimmer [36]. In all cases, the focus was on the limit of small advection and on the existence of a threshold. Here we give a general treatment and explore the advection-dominated regime. We first explain how to obtain a self-consistent relation on the velocity and present various analytical approximations for the solution. Second, we discuss the physical content of the results, focusing in particular on the size dependence of the velocity.

We consider the steady motion of a point source moving at constant velocity $\mathbf{v}$ and releasing at total rate $\mathcal{J}$ a quantity $\phi$ that may be a solute or heat. The spreading is controlled by a linear equation with Green function $G(\mathbf{r}, t)$. Denoting as $\mathbf{R}(t)$ the source position and using the notations of Fig. 1, the field in steady state is

$$
\phi(\mathbf{r})=\mathcal{J} \int_{-\infty}^{0} G(\mathbf{r}-\mathbf{R}(t),-t) d t .
$$

We will assume that the field evolution is governed by a diffusion process

$$
\partial_{t} \phi=D \Delta \phi+\mathcal{J} \delta(\mathbf{r}-\mathbf{R}(t))
$$




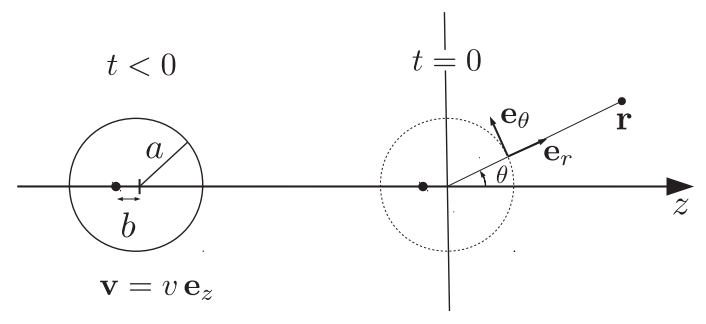

FIG. 1. Schematic of the swimmer investigated. The motion is steady with velocity $v$. The point source is shifted by a distance $b$ behind the center and has position $\mathbf{R}(t)=(v t-b) \mathbf{e}_{z}$. At time $t=0$, the swimmer center coincides with the origin.

with $D$ the diffusion coefficient and $\Delta$ the Laplacian. The Green function in dimension $d$ is then

$$
G(\mathbf{r}, t)=\frac{1}{(4 \pi D t)^{d / 2}} \exp \left[-\frac{\mathbf{r}^{2}}{4 D t}\right]
$$

From now on, we work in a frame of reference where the swimmer is fixed and the fluid velocity far from the swimmer is thus $-\mathbf{v}$. The concentration field is governed by the advection-diffusion equation,

$$
\mathbf{v} \cdot \nabla \phi+D \Delta \phi+\mathcal{J} \delta(\mathbf{r}-\mathbf{R}(0))=0
$$

In an exact treatment, the advecting velocity field would be the spatially dependent solution of the Navier-Stokes equation. Here the advecting flow is instead uniform, taking everywhere its far-field value which is simply fixed by the swimmer motion. Thus, the point-source model does account for advection of the chemical by the fluid but does so by introducing a drastically simplified flow field.

The swimmer is assumed to be a sphere, if phoretic, or a disk, if interfacial, with radius $a$. The point source is shifted a distance $b<a$ behind the center, with $b>0$ so that the swimmer moves forward. The swimmer is symmetric only if $b=0$. There are only three dimensionless parameters in the model. The first is the Péclet number that compares advection and diffusion, while the second is the $\chi$ ratio quantifying the geometric asymmetry,

$$
\mathrm{Pe}=\frac{a v}{2 D}, \quad \chi=\frac{b}{a} .
$$

A word of caution is required here: Our Péclet number is defined with respect to the real swimmer velocity, not the natural phoretic velocity as in some previous works, Ref. [35] in particular. Our notation for the Péclet number related to the natural velocity scale is actually the last parameter $M$, introduced below, whose definition depends on the situation considered. Even though there are several distinct expressions, we use the same symbol everywhere. Finally, note that the dimension we refer to below is associated with the field and is not the dimension of the swimmer.

\section{B. Applications}

\section{Three-dimensional phoretic swimmer}

Here the field $\phi$ is three-dimensional (3D) and its gradient induces on the surface of the swimmer a slip velocity,

$$
\mathbf{u}_{s}=\left.\frac{\mathcal{M}}{a} \partial_{\theta} \phi\right|_{r=a} \mathbf{e}_{\theta},
$$

where $\mathcal{M}$ is the phoretic mobility. For concreteness, the swimmer is assumed diffusiophoretic [57]. The field $\phi$ is then a solute concentration and the mobility, assumed constant for simplicity, is

$$
\mathcal{M}=\frac{k_{B} T \lambda^{2}}{\eta},
$$

with $k_{B}$ the Boltzmann constant, $T$ the temperature, $\eta$ the fluid viscosity, and $\lambda$ an effective interaction length that depends on the solid-liquid interaction potential. The resulting velocity is obtained from an average of the slip velocity over the surface $\mathcal{S}$ of the swimmer [8],

$$
\mathbf{v}=-\left\langle\mathbf{u}_{s}\right\rangle_{\mathcal{S}}=-\frac{1}{4 \pi a^{2}} \int_{\mathcal{S}} \mathbf{u}_{s} d S .
$$

With the notations of Fig. 1, the two above equations yield

$$
v=-\frac{\mathcal{M}}{2 a} \int_{0}^{\pi} \phi(a, \theta) \sin (2 \theta) d \theta .
$$

Now, for a symmetric swimmer, combining Eqs. (1)-(3) gives

$$
\phi(r, \theta)=\frac{\mathcal{J}}{4 \pi D r} \exp \left[-\frac{v r}{2 D}(1+\cos \theta)\right],
$$

thus leading to a closed equation for the velocity

$$
\frac{\mathrm{Pe}^{3}}{M}=e^{-\mathrm{Pe}}(\mathrm{Pe} \cosh \mathrm{Pe}-\sinh \mathrm{Pe}),
$$

where $M=\mathcal{M J} / 4 \pi a D^{2}$ can be seen as a Péclet number associated with the intrinsic phoretic velocity [58]. The above calculation can be extended to an asymmetric swimmer. With the notation $\Lambda=\sqrt{\mathrm{Pe} / 2 \chi}$, the result is

$$
\begin{aligned}
\frac{4 \chi \mathrm{Pe}^{2}}{M}= & e^{-2(1+\chi) \mathrm{Pe}}-1+2 \chi+\sqrt{\frac{\pi}{2 \chi \mathrm{Pe}}}(\chi-\mathrm{Pe}) \\
& \times e^{-\Lambda^{2}}\{\operatorname{erf}(\Lambda)-\operatorname{erf}[(1+2 \chi) \Lambda]\} .
\end{aligned}
$$

We now explore the solution of this self-consistent relation for the velocity. Though an analytical solution is not available in the general case, it can be obtained in the limit of small and large Péclet numbers. Some details of the calculations are provided in Appendix A. Using a small-Pe expansion, we find at lowest order

$$
\begin{aligned}
& \chi=0, \quad \mathrm{Pe}=\frac{M}{M_{c}}-1, \quad \text { for } M>M_{c}=3, \\
& \chi>0, \quad \mathrm{Pe}=\frac{\chi M}{3} .
\end{aligned}
$$

On the other hand, a large-Pe expansion yields, irrespective of $\chi$,

$$
\mathrm{Pe}=\sqrt{\frac{M}{2}}-\frac{1}{2}+\frac{3}{4} \sqrt{\frac{1}{2 M}}+\mathcal{O}\left(M^{-3 / 2}\right)
$$




\section{Three-dimensional interfacial swimmer}

We now consider a disk-shaped interfacial swimmer. For the sake of tractability, the dependence of the surface tension $\gamma$ on the tridimensional field $\phi$ is assumed linear, as quantified by the $\kappa$ parameter

$$
\gamma=\gamma_{o}-\kappa \phi
$$

Remembering that we consider only the steady motion, where the swimmer inertia plays no role, the velocity is fixed by the balance between the surface tension resultant and the drag force,

$$
F_{s}=-2 \kappa a \int_{0}^{\pi} \phi(a, \theta) \cos (\theta) d \theta=F_{d} .
$$

In the same spirit as above, we again decouple the concentration and flow problems by evaluating $F_{d}$ as the drag experienced by the swimmer in the absence of chemical release. Besides, throughout Sec. II, the drag is given explicitly by the Stokes expression $F_{d}=C \eta a v$, with $C$ a numerical prefactor that depends only on the swimmer shape. Such a choice permits a unified treatment and an exhaustive comparison of the phoretic and interfacial swimmers. However, it is not intrinsic to the point-source model and can be relaxed, as discussed in Sec. III C.

If the field is a chemical (or heat) diffusing within the three-dimensional half-space below the swimmer [59], then retracing the steps above leads to

$$
\frac{\mathrm{Pe}}{M}=-\int_{0}^{\pi} \frac{\cos \theta}{2 \pi \tilde{r}} \exp [-\mathrm{Pe}(\tilde{r}+\cos \theta+\chi)] d \theta,
$$

with $\tilde{r}=\sqrt{1+2 \chi \cos \theta+\chi^{2}}$ and $M=\kappa \mathcal{J} / C \eta D^{2}$, which may again be interpreted as the Péclet number associated with the natural velocity scale [60]. For a symmetric swimmer, this simplifies into $2 \mathrm{Pe} / M=\exp (-\mathrm{Pe}) I_{1}(\mathrm{Pe})$, where $I_{1}$ is the modified Bessel function of the first kind and order one. In the presence of asymmetry, we could not evaluate the integral in closed form but we can nevertheless investigate the solution in limiting cases. In the regime of small Péclet number, we find at lowest order,

$$
\begin{aligned}
& \chi=0, \quad \mathrm{Pe}=\frac{M}{M_{c}}-1, \quad \text { for } M>M_{c}=4, \\
& \chi>0, \quad \mathrm{Pe}=\frac{\chi M}{4} .
\end{aligned}
$$

For large Pe, the integral in Eq. (18) can be evaluated using the Laplace method, with the result

$$
\mathrm{Pe}=\frac{M^{2 / 3}}{2[\pi(1-\chi)]^{1 / 3}} .
$$

\section{Two-dimensional phoretic or interfacial swimmer}

Here the system may be either an interfacial swimmer where the surfactant released is insoluble and thus remains at the air-liquid interface or a truly two-dimensional phoretic swimmer, as considered in Refs. [34,61]. Both cases lead to the same equation

$$
\frac{\mathrm{Pe}}{M}=-\int_{0}^{\pi} \frac{\cos \theta}{2 \pi} e^{-\mathrm{Pe}(\cos \theta+\chi)} K_{0}(\tilde{r} \mathrm{Pe}) d \theta,
$$

with $\tilde{r}$ defined as in Eq. (18), $K_{0}$ the modified Bessel function of the second kind, and $M=\kappa \mathcal{J a} / C \eta D^{2}$ and $M=\mathcal{M} \mathcal{J} / 4 \pi D^{2}$ for the interfacial and phoretic swimmers, respectively. The behavior at small $\mathrm{Pe}$ is now

$$
\begin{aligned}
& \chi=0, \quad \mathrm{Pe}=2 e^{-4 / M-\gamma_{\mathrm{EM}},} \\
& \chi>0, \quad \mathrm{Pe}=\frac{\chi M}{4},
\end{aligned}
$$

with $\gamma_{\text {EM }}$ the Euler-Mascheroni constant. For large Pe, the Laplace method yields

$$
\mathrm{Pe}=\frac{\sqrt{M}}{2}-\frac{1}{4}+\mathcal{O}\left(M^{-1 / 2}\right) .
$$

Finally, in the case of interfacial swimmers, the model may be extended to account for evaporation. If the insoluble surfactant disappears from the surface at rate $\alpha$, then the concentration equation becomes $\partial_{t} \phi=D \Delta \phi-\alpha \phi+\mathcal{J} \delta(\mathbf{r}-\mathbf{R}(t))$, and the solution now includes a factor $e^{-\alpha t}$. Considering the symmetric swimmer only, the equation for velocity now reads as

$$
\frac{2 \mathrm{Pe}}{M}=e^{-\chi \mathrm{Pe}} I_{1}(\mathrm{Pe}) K_{0}\left(\sqrt{\mathrm{Pe}^{2}+A^{2}}\right),
$$

where $A^{2}=a^{2} \alpha / D$ is the ratio of typical diffusion and evaporation times. The behavior at small velocities is qualitatively changed,

$$
\mathrm{Pe}=\sqrt{M-M_{c}}, \quad \text { for } M>M_{c}=\frac{1}{K_{0}(A)} .
$$

The large-Pe behavior is not modified at lowest order: The term $-1 / 4$ in Eq. (25) is replaced with $-\left(1+A^{2}\right) / 4$.

A full numerical solution for the $\operatorname{Pe}(M)$ relation is shown in Fig. 2 for all cases, along with the approximations developed.

\section{Discussion}

We now discuss the results above, focusing on the velocity. To obtain specific predictions, one needs to specify how the total release rate varies with the swimmer size. The most natural choice at this point is $\mathcal{J}=\pi(2 a)^{d^{\prime}-1} J$, with $J$ a lineic or surfacic flux, for a swimmer dimension $d^{\prime}=2$ or 3 , respectively. The resulting formulas for the velocity are shown in Table I.

Consider first the low-Pe regime. For an asymmetric swimmer $(\chi>0)$, the velocity, up to a numerical prefactor, is

$$
v \simeq \frac{\chi \mu J}{D} a^{\varepsilon}
$$

with $\mu$ akin to a mobility (see Table I) and the exponent $\varepsilon=0$ and 1 for phoretic and interfacial swimmer, respectively. For the latter, the velocity scales linearly with size, suggesting that this mechanism is not robust against downsizing. In contrast, the velocity of a phoretic swimmer is proportional to asymmetry and chemical activity but independent of size. Notably, these dependencies precisely correspond to what has been found with more accurate approaches [31,61], indicating that the point-source model, despite its various simplifying assumptions, successfully captures the physics at play.

For the symmetric swimmer, exact results are available in the 3D autophoretic case [35]. Symmetry breaking occurs only above a threshold value and the function $2 \mathrm{Pe} / M$ 

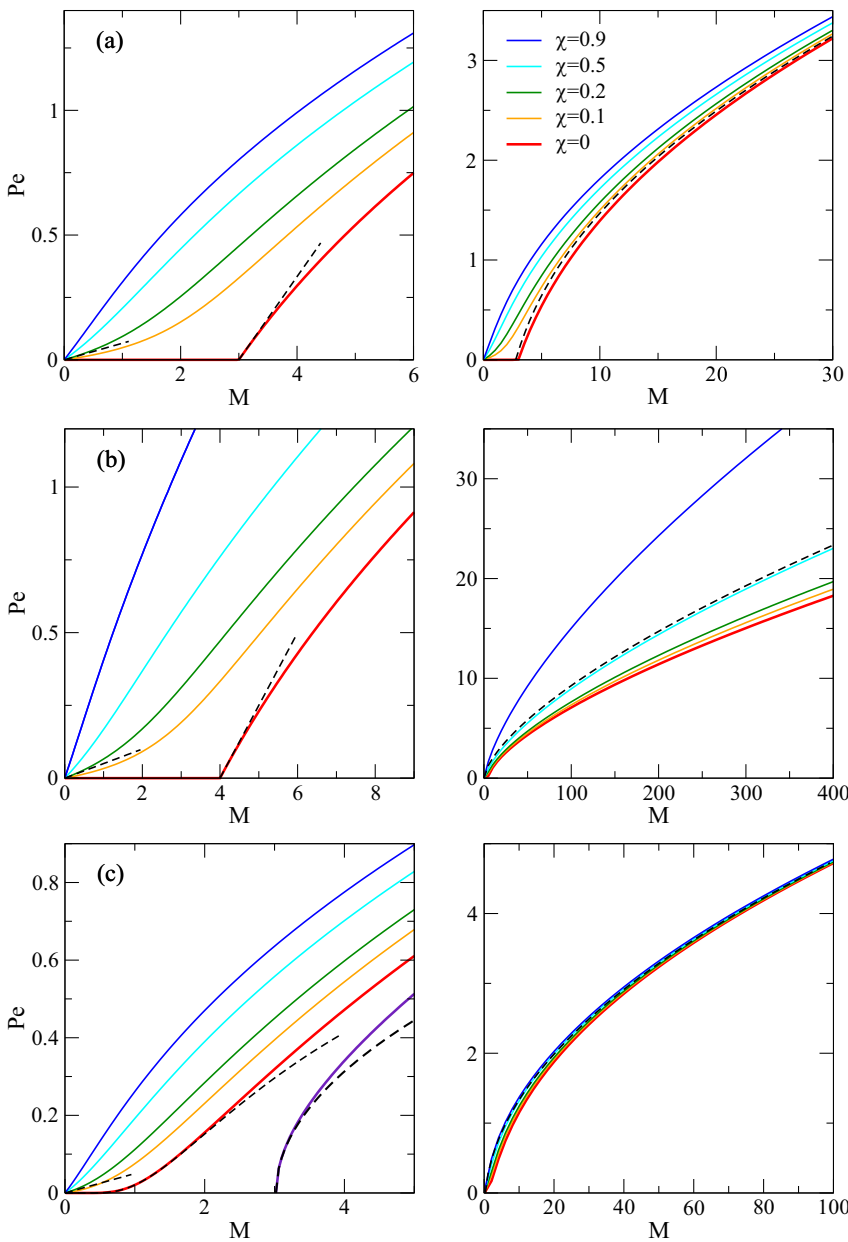

FIG. 2. $\operatorname{Pe}(M)$ relation in the point-source model in the small and large Pe regimes (left and right, respectively) for 3D autophoretic (a), 3D interfacial (b), and 2D swimmers (c). Full lines give the solution computed numerically for asymmetry parameter $\chi=0,0.1,0.2,0.5$, and 0.9 , from bottom to top. The purple line is the $2 \mathrm{D}$ symmetric case with evaporation and $A^{2}=0.1$. Approximations are shown in dashed lines. Going from left to right, they correspond to (a) Eqs. (14), (13), and (15); (b) Eqs. (20), (19), and (21) with $\chi=0.5$; and (c) Eqs. (24), (23), (27), and (25).

computed numerically exhibits a maximum followed by a gentle decay. Our model yields a similar behavior. Quantitatively, the exact threshold is $M_{c}=4$, we find 3 . The maximum occurs at $M^{*} \simeq 9$ with magnitude $2 \mathrm{Pe}^{*} / M^{*} \simeq 0.08$, we find $M^{*} \simeq 9.6$ and $2 \mathrm{Pe}^{*} / M^{*} \simeq 0.28$. The point-source model thus overestimates the velocity magnitude by a factor of three but reproduces all noticeable features-velocity threshold and maximum - for which it provides reasonable estimates. The overestimation of velocity can be chiefly ascribed to the neglect of the swimmer excluded volume. As explained in Sec. A of the Supplemental Material (S.M. [62]), a simple extension of the point-source model that partially accounts for this effect leads to much better predictions for the velocity. It remains that the qualitative features of autophoretic behaviors are correctly reproduced by the point-source model, suggesting that it can indeed provide significant insight into the self-propulsion mechanism.
TABLE I. Velocity predicted by the point-source model as a function of swimmer type, regime, and field dimension. The symbol $\mu$ is defined as $\mathcal{M} / 4 \pi$ and $\kappa / C \eta$ for the phoretic and interfacial swimmers, respectively. Numerical prefactors are discarded. Here we assume $\mathcal{J} \sim a^{d^{\prime}-1} J$. Only the asymmetric case is reported (see the text for symmetric swimmers).

\begin{tabular}{lccccc}
\hline \hline \multirow{2}{*}{$\begin{array}{l}\text { Regime } \\
\text { swimmer }\end{array}$} & \multicolumn{2}{c}{ Low Pe } & & \multicolumn{2}{c}{ High Pe } \\
\cline { 2 - 3 } \cline { 5 - 6 } & Phoretic & Interfacial & & Phoretic & Interfacial \\
\hline 2D & $\frac{\chi \mu J}{D}$ & $\frac{\chi \mu J a}{D}$ & & $\left(\frac{\mu J}{a}\right)^{1 / 2}$ & $(\mu J)^{1 / 2}$ \\
3D & $\prime \prime$ & $\prime \prime$ & & $\prime \prime$ & $\left(\frac{\mu^{2} J^{2} a}{D(1-\chi)}\right)^{1 / 3}$ \\
\hline \hline
\end{tabular}

The spontaneous motion by symmetry breaking involves a threshold in several cases: For both types of swimmer if the field is $3 \mathrm{D}$, and for an interfacial swimmer with $2 \mathrm{D}$ field in the presence of evaporation. The bifurcation is always continuous (no jump) but slightly above the threshold, the increase in velocity may be linear or involves a square-root singularity [Eqs. (19) and (27), respectively]. When the evaporation rate approaches zero, the threshold vanishes. The velocity is nonzero but is exponentially small in $1 / M$, implying that motion is spontaneous albeit very slow. If the existence of a threshold is a notable feature of the model, then its detection may be an experimental challenge. The slightest defect, which can hardly be avoided, may induce asymmetry and make the threshold irrelevant. We thus expect that at low Pe, the behavior of real systems is dominated by intrinsic asymmetry.

The situation is different in the high-Pe regime. In three cases of four (Table I), asymmetry does not appear at lowest order. Here the effect of advection dominates. Distinct behaviors are observed for the size dependence of the velocity. For phoretic swimmers, the velocity would decrease as $a^{-1 / 2}$, a trend a priori not relevant to real systems where the highPe regime seems hardly accessible, as explained below. For an interfacial swimmer with 2D field, the velocity is size independent. In contrast, larger 3D swimmers move faster, with $v \sim a^{1 / 3}$. Note that different dependencies may be found if the assumption $\mathcal{J} \sim a^{d^{\prime}-1} J$ is relaxed.

The 3D interfacial swimmer, the fourth case in the high-Pe regime, is peculiar in that asymmetry still matters. This difference can be rationalized by a simple argument. Because advection is dominant, the chemical is found mostly behind the swimmer, in a thin wake of width $w=\sqrt{D \Delta t}$, where $\Delta t=$ $a(1-\chi) / v$ is the advection time from the release point to the periphery. The balance between drag and surface tension forces yields $C \eta a v=\phi w \kappa$, with $\phi=\mathcal{J} / v w^{d-1}$ the typical value of concentration [63]. In 2D, one obtains $v^{2}=\kappa \mathcal{J} / C \eta a$ and $\mathrm{Pe} \sim M^{1 / 2}$. Consistent with Eq. (25), the diffusion and asymmetry are irrelevant. In the 3D case, one finds instead $\mathrm{Pe} \sim M^{2 / 3} /(1-\chi)^{1 / 3}$. The (small) spreading of the chemical in the lateral direction has no influence on the velocity as in the $2 \mathrm{D}$ case, but spreading in the depth of the liquid reduces the chemical concentration at the surface. As a result, the exponent is different and the influence of asymmetry is retained.

Having analyzed the low- and high-Pe limits, we finally examine orders of magnitude of $M$ to see which regime is relevant to each type of swimmer. To estimate $M_{\mathrm{pho}, 3 \mathrm{D}}$ for 
a 3D phoretic swimmer, we use Eq. (7) for the phoretic mobility with $k_{B} T=410^{-21} \mathrm{~J}, \lambda=10^{-9} \mathrm{~m}, \eta=10^{-3} \mathrm{~Pa} \mathrm{~s}$, and $D=10^{-9} \mathrm{~m}^{2} \mathrm{~s}^{-1}$. For $J=\mathcal{N}_{A} \mathrm{~m}^{-2} \mathrm{~s}^{-1}$ [36], where $\mathcal{N}_{A}$ is Avogadro's number, we find a value of order unity. Since our $J$ value is probably an upper estimate [64], $M_{\text {pho,3D }}$ is generally smaller than unity. Now, assuming for comparison purpose that the (surface) chemical activity $J$ is the same for the phoretic and interfacial swimmer, we can obtain for the ratio of their $M$ numbers a simple expression that involves only characteristic length scales,

$$
\frac{M_{\mathrm{int}, 3 \mathrm{D}}}{M_{\mathrm{pho}, 3 \mathrm{D}}}=\frac{l_{\mathrm{ads}}}{\lambda^{2}} \frac{a_{\mathrm{int}}^{2}}{a_{\mathrm{pho}}} .
$$

Here subscripts indicate the type of swimmer and the adsorption length $l_{\mathrm{ads}}$ is the ratio between the surface and bulk concentration of the chemical [65]. Using $a_{\text {int }}=10^{-2} \mathrm{~m}$, $a_{\text {pho }}=10^{-6} \mathrm{~m}$, and $l_{\text {ads }} \simeq 10^{-6} \mathrm{~m} \mathrm{[66],} \mathrm{we} \mathrm{see} \mathrm{that} \mathrm{for} \lambda$ ranging from angströms to microns, the ratio $M_{\text {int,3D }} / M_{\text {pho,3D }}$ is a very large number, many orders of magnitude above unity.

As a consequence, the phoretic and interfacial swimmers operate in very different regimes. In the former, $M$ is small, generally below unity, and so is the Péclet number $\mathrm{Pe} \sim M$. The propulsion is dominated by asymmetry and the symmetry-breaking mechanism cannot operate. This may explain why no experimental instance has been reported so far. In contrast, interfacial swimmers are characterized by a very large $M$. Here advection dominates and the intrinsic asymmetry plays only a minor role, if any. The relation $\mathrm{Pe} \sim M^{v}$ is sublinear $(v<1)$. To put these conclusions to test, we now turn to experiments.

\section{EXPERIMENTS ON CAMPHOR DISKS}

\section{A. Protocol and parameters}

\section{Swimmer fabrication and set-up}

Building on previous studies $[29,67]$, the fabrication protocol of our camphor swimmers involves several steps. A sheet of agar gel is first produced from a $5 \% \mathrm{w} / \mathrm{w}$ aqueous solution of agarose. To obtain the desired thickness of $0.5 \mathrm{~mm}$, the melted gel is poured on a plate and quickly spread with a metal bar. The agar sheet is soaked subsequently in three solutions: (i) for at least $2 \mathrm{~h}$ in a methanol solution so that the water trapped in the gel is replaced by methanol, (ii) for $12 \mathrm{~h}$ in a methanol solution saturated with camphor $\left(1.1 \mathrm{~g} \mathrm{ml}^{-1}\right.$ [68]), and (iii) for at least $30 \mathrm{~s}$ in water saturated with camphor $\left(1.2 \mathrm{~g} \mathrm{l}^{-1}\right.$ [68]) twice. At this point, the substitution of methanol by water induces the precipitation of camphor and the sheets turn opaque and white. For brevity, such loaded agar gel is henceforth referred to as the solid. If not used immediately, then the prepared sheets are preserved in the last bath, for a maximum duration of one day. Circular disks with radius $a$ ranging from 1 to $9 \mathrm{~mm}$ are cut with punchers. For larger disks, a computer-controlled cutter is used on the agar gel prior to the loading bath.

A single camphor disk is gently placed at the water surface so as to avoid submersion and its motion is recorded with a digital video camera [69] during a run time of typically $15 \mathrm{~min}$. The swimmer trajectories are reconstructed using a homemade tracking code based on Crocker-Grier algorithm

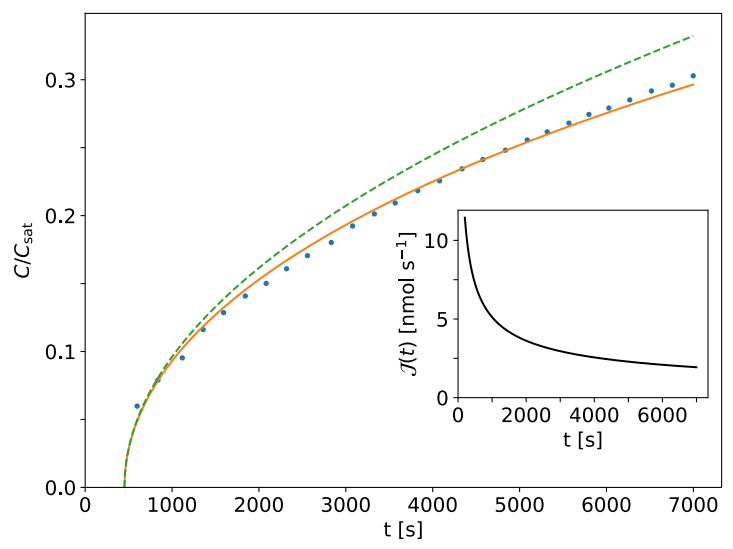

FIG. 3. Measurement of camphor release by 25 fully immersed 4-mm camphor disks (see Sec. C of S.M. [62] for details). $C(t)$ is the concentration in the solution at time $t$. The continuous line is a fit to Eq. (31) including a time shift $t_{o}=450 \mathrm{~s}$ and giving a characteristic time $\tau=1.2 \times 10^{5} \mathrm{~s}$. The dashed line is the concentration predicted if camphor does not accumulate in the solution $\left(C(t) \ll C_{\text {sat }}\right)$, as given by Eq. (32). (Inset) Expected flux for a single swimmer as a function of time.

and circle recognition [70,71]. Unless otherwise mentioned, the pool is rectangular with dimensions $27 \times 17 \times 1 \mathrm{~cm}$, made of Plexiglas, and filled with millipore water. An essential precaution is to avoid contamination by any external agent such as dust. Accordingly, the whole set-up is covered by a Plexiglas box. After each run, the water is sucked at the surface and replaced with clean water. Every four runs, the pool solution is totally renewed. As explained in Sec. B of S.M. [62], the camphor accumulated in solution has no detectable influence on the swimmer velocity.

\section{Camphor release}

The estimation of parameters involved in the swimming of camphor disks is far from obvious [56]. One crucial quantity is the total release rate $\mathcal{J}(t)$ from a single swimmer, a number that may change with time. As detailed in Sec. C of S.M. [62], we used spectrometry to measure the amount of camphor released by swimmers $4 \mathrm{~mm}$ in radius when totally immersed in a fixed volume of water. The time dependence of the camphor concentration is shown in Fig. 3.

A simple model of camphor release is proposed to interpret these data. The basic idea is that the dissolution is controlled by a growing layer where the camphor is no more crystallized but diffusing through hydrogel water. As illustrated in Fig. 4, the camphor concentration is $C_{\text {sol }}$ in the solid domain, $C_{\text {sat }}$ at its boundary, and $C(t)$ everywhere in the volume $V$ of solution outside, assumed well mixed. The phenomenon is approximated as one-dimensional and occurring over the total surface area $\mathcal{A}$ of the swimmer. To obtain the thickness $\delta(t)$ of the growing layer, the total flux is written in two ways,

$$
\frac{\mathcal{J}(t)}{\mathcal{A}}=C_{\mathrm{sol}} \dot{\delta}(t)=D_{g} \frac{C_{\mathrm{sat}}-C(t)}{\delta(t)},
$$

with $D_{g}$ the diffusion coefficient in the gel. The former equation describes the camphor released by a front advancing at velocity $\dot{\delta}(t)$ in the solid, while the latter is the diffusion 


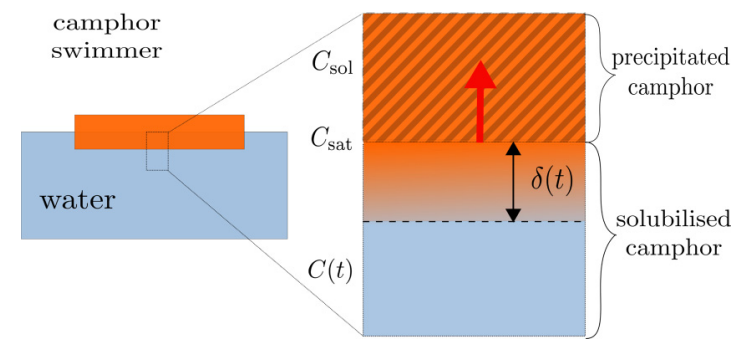

FIG. 4. Growing diffusive layer model. The transport of camphor is governed by diffusion in the gel layer and an advancing front that moves in the solid domain. Notations are introduced in the text.

flux induced by a constant gradient estimated in a quasisteady approximation. Together with $\mathcal{J}(t)=V \dot{C}(t)$, Eq. (30) yields a closed system whose solution is

$$
\frac{C(t)}{C_{\mathrm{sat}}}=1+W[-\exp (-t / \tau-1)] .
$$

Here $W$ is the Lambert function, the solution of $W(x) \exp [W(x)]=x$, and $\tau=V^{2} C_{\text {sat }} / \mathcal{A}^{2} D_{g} C_{\text {sol }}$ is a characteristic time. In the special case where the liquid volume is very large and $C(t)$ remains negligible with respect to $C_{\text {sat }}$, the solution simplifies to

$$
\frac{C(t)}{C_{\mathrm{sat}}}=\sqrt{2 t / \tau},
$$

which also provides a good approximation of the full solution at early time $(t \ll \tau)$.

As visible in Fig. 3, the growing diffusive layer model gives a satisfactory description of the concentration in the release experiment, provided we allow for a shift in time origin of approximately $t_{o}=450 \mathrm{~s}$. Several factors might be suggested as an explanation. First, the model is only approximate at the earliest time, where the flux diverges. Second, a time of about $2 \mathrm{~min}$ is needed to prepare the 25 camphor disks, which are not immersed simultaneously and may lose some camphor by sublimation. Finally, the early release might be influenced by the initial state of the surface. In any case, the time shift remains small compared to the characteristic time. From the latter, one can deduce a diffusion coefficient in the gel $D_{g}=0.4 \times 10^{-9} \mathrm{~m}^{2} \mathrm{~s}^{-1}$, which is close and below the value in free water (see Table II). Note also that at the end of the release measurement, the concentration $C(t)$ is a significant fraction of $C_{\mathrm{sat}}$. In experiments involving a single camphor disk in the pool, such an accumulation is not expected because only one half-immersed swimmer is used, rather than 25 totally immersed disks, for a liquid volume which is roughly the same. Furthermore, camphor is free to evaporate from the water surface, thus providing a self-cleaning mechanism. We thus expect Eq. (32) to appropriately describe the release in the swimming situation. Besides, while the water is not stirred in the swimming set-up, the disk motion is probably sufficient to avoid local accumulation. Finally, the transport of camphor toward the air-water interface might involve two channels: dissolution from the immersed face of the disk or sublimation from the dry face. Whereas the latter is known to exist with solid camphor [72], we assume here the former is dominant because in our system camphor is embedded in an aqueous gel. Keeping in mind that only one face of a swimming disk is immersed, the expected flux for a single swimmer is plotted in the inset of Fig. 3. For convenience, all parameters and their numerical values have been listed in Table II.

\section{B. Results}

Although the focus so far has been on the steady velocity of a swimmer in an infinite medium, experiments indicate that the velocity of a swimmer may change with time and pool depth and in the vicinity of pool boundaries. We first describe these dependencies and specify how the velocities discussed below are obtained. As explained in Sec. D of S.M. [62], swimmers slow down near the walls, with a velocity reduced whenever the distance to the wall is less than six swimmer radii. To avoid such boundary effects, all velocities shown below are computed discarding trajectory points within this exclusion zone.

A camphor disk may swim for hours but its velocity is time dependent as illustrated in Fig. 5. In addition to rapid fluctuations and intermittent interruption when the swimmer is transiently blocked at the wall, the velocity exhibits a slow decrease with time, dropping by a factor of three over the course of a 4-h experiment. Such an evolution is expected from the decrease in release rate but could also involve changes in the water-subphase properties. This circumstance, however, is unlikely to apply because, as described above, the pollution of the subphase by camphor release is negligible. As detailed in Sec. B of S.M. [62], complementary experiments involving swimmers released at successive times in the same pool confirm that the velocity evolution entirely reflects intrinsic properties of swimmers.

A steady-state approximation applies best at long times when the rate of change in velocity is the smallest, but we

\begin{tabular}{|c|c|c|c|}
\hline Quantity & Symbol & Value & Source \\
\hline Swimmer radius and thickness & $a, c$ & $1-15,0.5 \mathrm{~mm}$ & - \\
\hline Drag factor for a disk moving edgewise at interface & $C$ & $16 / 3$ & [73] \\
\hline Camphor concentration at saturation and in solid & $C_{\mathrm{sat}}, C_{\mathrm{sol}}$ & $7.9,5200 \mathrm{~mol} \mathrm{~m}^{-3}$ & {$[62,68]$} \\
\hline Diffusion coefficient of camphor in water & $D$ & $0.72 \times 10^{-9} \mathrm{~m}^{2} \mathrm{~s}^{-1}$ & [74] \\
\hline Release rate for a single swimmer ${ }^{\mathrm{a}}$ & $\mathcal{J}$ & $5 \times 10^{-9} \mathrm{~mol} \mathrm{~s}^{-1}$ & Fig. 3 \\
\hline Dynamic viscosity of water & $\eta$ & $1 \times 10^{-3} \mathrm{~Pa} \mathrm{~s}$ & - \\
\hline Concentration dependence of surface tension [Eq. (16)] & $\kappa$ & $3 \times 10^{-3} \mathrm{~N} \mathrm{~m}^{2} \mathrm{~mol}^{-1}$ & [67] \\
\hline
\end{tabular}

TABLE II. Numerical value of parameters used in this study.

${ }^{\mathrm{a} \mathcal{J}}$ is the total release rate for a 4-mm swimmer half-immersed and estimated from the inset of Fig. 3 at time $t=800 \mathrm{~s}$ (see Sec. III A 2). 


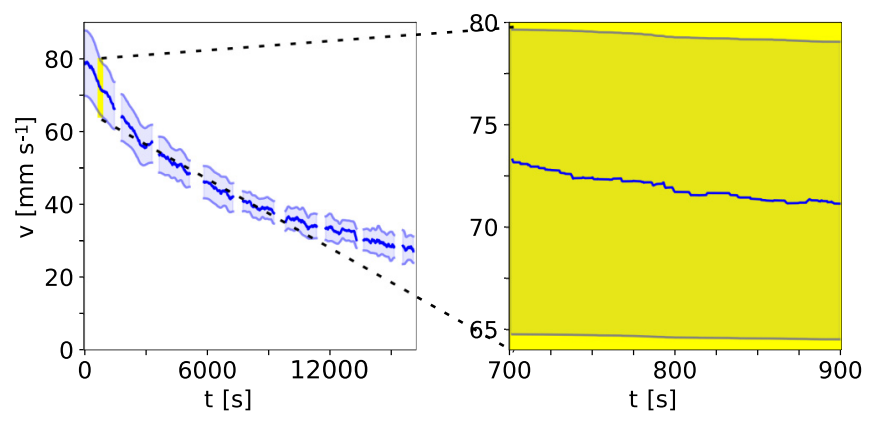

FIG. 5. Time dependence of the velocity. The swimmer has a radius $a=4 \mathrm{~mm}$. Left: The line is the data averaged over a 1-min sliding window and the blue area around corresponds to one standard deviation. Right: Close-up on the 700- to 900-s interval over which the velocity is computed. Note that the average value decreases by only a few percent.

have found it more convenient to focus on the intermediate regime because shorter experiments are more easily repeated. For this reason, the velocity of a given swimmer is the value time averaged over the $700-$ to $900-$ s interval. For a given set of parameters, typically three or four different swimmers, usually cut from the same camphor-loaded gel sheet, are observed. The velocities values reported below results from an average over those independent realizations and the error bars correspond to a $95 \%$ confidence interval obtained from Student's law.

The velocity of camphor boats has been shown to be strongly dependent on depth in a one-dimensional pool, wherein the velocity may actually vanish [48]. Shown in Fig. 6 is the velocity dependence on pool depth $h$ for our camphor disks, measured in a different, deeper pool [75]. Within error bars, the velocity is independent of pool depth for values exceeding two or three times the swimmer radius. Whereas the shallowest pool tested $(h / a \simeq 0.6)$ leads to a clear reduction in velocity, we note that already for $h / a$ around unity, the measured value is a reasonable approximation of the infinite-depth velocity.

Having specified the measurement of velocities, we can now proceed to the main focus of this work: The influence of asymmetry and size. In contrast to the other parameters,

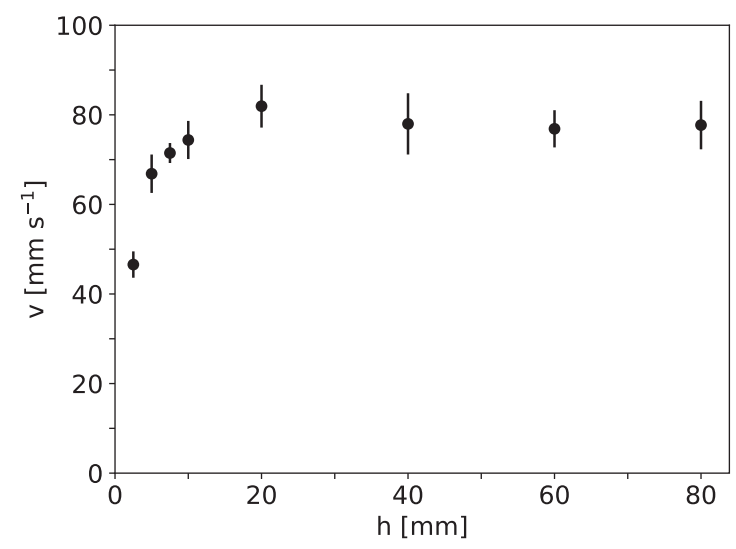

FIG. 6. Depth dependence of the velocity. The swimmers are $4 \mathrm{~mm}$ in radius.

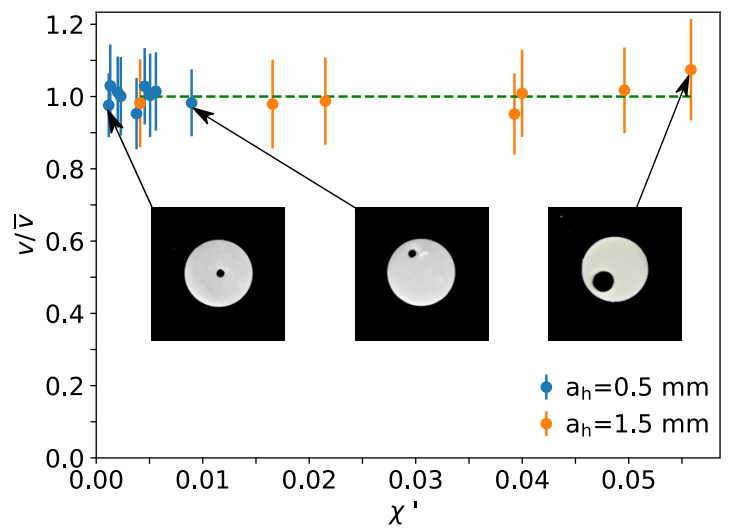

FIG. 7. Swimming velocity of camphor disks with controlled asymmetry. For each hole size $a_{\mathrm{h}}$, the velocity is normalized by the average value $\bar{v}$. The dashed line corresponds to a unit ratio $v / \bar{v}=1$. A few swimmers are shown as insets. In all cases, $a=4 \mathrm{~mm}$ and $h=10 \mathrm{~mm}$.

both quantities are easily tuned experimentally. As regards asymmetry, it would be difficult to quantify how much our fabricated swimmers deviate from their ideal prescription in terms of shape and homogeneity. It is much easier to introduce a controlled asymmetry. To do so, the radial symmetry of the swimmer is broken by punching in the disk a small hole with radius $a_{\mathrm{h}}$ at a distance $b_{\mathrm{h}}$ from the disk center. Assuming camphor flux is uniform on the surface of the swimmer, the barycenter of camphor release is thus shifted by a distance $b^{\prime}=b_{\mathrm{h}}\left(a_{\mathrm{h}} / a\right)^{2}$ and we use $\chi^{\prime}=\left|b^{\prime} / a\right|$ to quantify this geometric asymmetry.

Figure 7 presents the velocity measured for swimmers with radius $4 \mathrm{~mm}$ and two hole sizes $a_{\mathrm{h}}=0.5$ and $1.5 \mathrm{~mm}$. Strikingly, within error bars, the influence of hole position is not detectable. The velocity is thus independent of asymmetry. This is a strong indication that the swimming velocity is governed not by intrinsic asymmetry but by a symmetry-breaking mechanism. Another implication is that the swimming velocity is a reproducible observable. Whereas each fabricated swimmer is probably unique, featuring its own defects, the velocity is not sensitive to these differences. We note that the asymmetry independence of velocity is fully consistent with previous observations on camphor boats [45]. In such a system, the camphor disk is attached below a rectangular plastic plate, at a fixed distance $d$ behind the plate center. There is a significant range of $d$ where propulsion is steady and the velocity remains constant, suggesting that the symmetrybreaking mechanism is at play here, too [76]. Finally, it is noteworthy that for small $d$, the camphor boat motion is intermittent but the maximum velocity is still independent of intrinsic asymmetry.

Our second main observation is that bigger swimmers move faster. This is shown in Fig. 8, where we report the velocity of swimmers with size ranging from 1 to $15 \mathrm{~mm}$. Before we comment on the curve, several remarks are in order. Swimmers with a radius above $15 \mathrm{~mm}$ were all partially sinking in water, which presumably induces a significant reduction of their velocity. Accordingly, they are not considered here. For the large swimmers $(a>9 \mathrm{~mm})$, a pool bigger than the default one was used. As regards the pool 


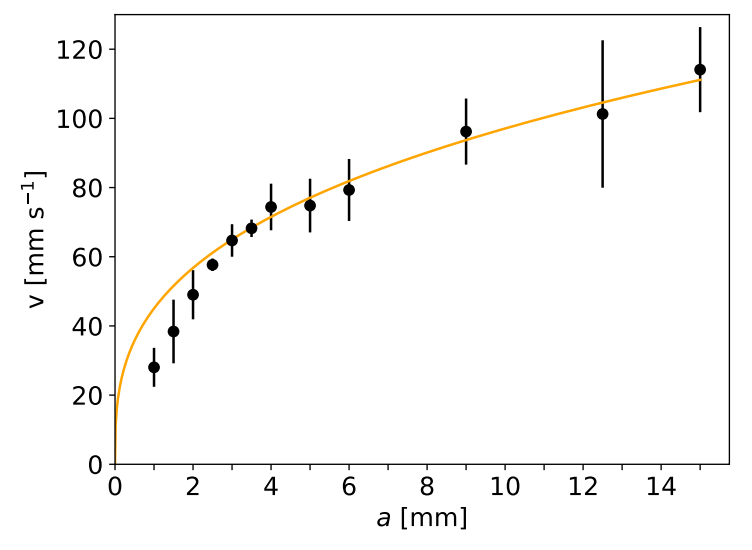

FIG. 8. Size dependence of the swimming velocity. The pool depth is $h=10 \mathrm{~mm}$. The continuous line is a fit to $v \sim a^{1 / 3}$.

depth, it is always fixed to $10 \mathrm{~mm}$. Although the velocity of large swimmers is affected by the finite depth, we found that the cleaning and replacement of water in deeper pools was impractical because of the large volume involved. Finally, it is natural in the theoretical analysis to use the dimensionless numbers Pe and $M$. The results of Fig. 8 could be recast as a $\operatorname{Pe}(M)$ relationship, but only by introducing some modeling assumptions, such as the size dependence of flux. To avoid any uncertainty associated with such steps, we prefer to report the experimental data as obtained. Coming back to the results, the measured velocities range from 25 to $140 \mathrm{~mm} \mathrm{~s}^{-1}$. Except for the smallest swimmers, the data are reasonably well described by a power law with exponent $1 / 3$. The dependence on swimmer size is thus clearly sublinear.

\section{Discussion}

We are finally in a position to discuss the predictions of the point-source model. The 3D (respectively, 2D) model posits that the chemical species released can be found only in the bulk (respectively, at the interface). Because camphor is a soluble surfactant present in both media, we will consider the two cases. Consistent with the growing diffusive layer model, we assume throughout that the release rate $\mathcal{J}$ is proportional to the swimmer area $\mathcal{J} \sim a^{2}$ [77]. Let us fix the radius to $a=4 \mathrm{~mm}$, the case most studied above, and start with an order-of-magnitude estimate for the $M$ number. Using parameters of Table II and the order of magnitude $\kappa=k_{B} T$ in $2 \mathrm{D}$, we find $M_{2 \mathrm{D}} \simeq 10^{13}$ and $M_{3 \mathrm{D}} \simeq 5 \times 10^{9}$. Both values are very large, far above any threshold, indicating that the large-Pe regime is the relevant one and that the asymptotic approximations given by Eqs. (25) and (21) are valid. Though $M_{2 \mathrm{D}}$ and $M_{3 \mathrm{D}}$ differ by several orders of magnitude, they lead to close estimates for the velocities: $v_{2 \mathrm{D}} \simeq 800$ and $v_{3 \mathrm{D}} \simeq$ $400 \mathrm{~mm} \mathrm{~s}^{-1}$. The observed value is $40 \mathrm{~mm} \mathrm{~s}^{-1}$. As in the diffusiophoretic case, the velocities are overestimated but the prediction remains a reasonable one.

Turning now to the parameter dependence of velocity, there are two main expectations from the model (i) asymmetry has no influence in the 2D case and only a small effect in 3D for weak asymmetry [78] and (ii) velocity should increase with swimmer size but sublinearly. Both points are in full agreement with experiments, as demonstrated by Figs. 7 and 8 .
Note the complete contrast with real phoretic swimmers, whose velocity is proportional to asymmetry and independent of their size. At a quantitative level, the model yields a power-law dependence $v \sim a^{v}$ with exponent $v=1 / 2$ and $1 / 3$ for the $2 \mathrm{D}$ and $3 \mathrm{D}$ cases, respectively, the latter being more compatible with data. As we discuss below, such an agreement should be considered with caution in view of the model approximations.

Overall, the predictions of the point-source model appear to be semiquantitative, a somewhat unexpected achievement given the simplifications involved. Before concluding, we discuss for our specific experimental system the model assumptions and we order them tentatively starting with the most important first. Throughout the discussion we take the swimmer with radius $4 \mathrm{~mm}$ as the reference case.

(i) Neglect of Marangoni flows. The inhomogeneous distribution of camphor at the interface induces a driving force on the swimming disk but also engenders so-called Marangoni flows that are not accounted for in the model yet may affect both the drag and driving forces. As demonstrated in a recent work [79], a point source releasing surfactant at a constant rate generates a velocity field at the interface given by $\left([\kappa \mathcal{J} /(2 \pi \eta)]^{2} v / r^{3}\right)^{1 / 5}$, with $r$ the distance from the source and $v$ the kinematic viscosity and the $2 \mathrm{D}$ case is assumed. In the spirit of the treatment above, we can evaluate this solution in the vicinity of the swimmer, which gives $140 \mathrm{~mm} \mathrm{~s}^{-1}$ for our reference case, a value comparable to the swimming velocity. Of course, the presence of a solid disk rather than a punctual source will reduce the magnitude of this effect. Yet Marangoni flows appear as one missing physical ingredient potentially important. Qualitatively, they will tend to oppose the formation of surface concentration gradients. It is thus likely that the neglect of Marangoni flows in the point-source model is one significant factor in the overestimation of velocity. To date, however, it remains a challenge to account for them in a simple manner and it will certainly require future investigations. Note that Refs. [80,81] suggest that in some situations the outcome of Marangoni flows is to renormalize the effective diffusivity of the surfactant. If applicable in our system, then such an equivalence would offer a very simple path to account for Marangoni effects within the point-source framework.

(ii) Stokes drag. While this assumption permits a unified treatment of phoretic and interfacial swimmers, it is not accurate for large interfacial swimmers, since their Reynolds number can be well above unity. Numerical simulations for the reference case indicate that the drag on a disk moving edgewise at the swimmer velocity is five times higher than the Stokes value, thus pointing to another possible cause for the overestimation of velocity. An even stronger drag force can be expected if one accounts for the deformation of interface, most relevant for larger sizes, and the finite thickness of the swimmers, most important for smaller sizes. We note that assuming a viscous boundary layer [82] under the disk leads to a drag force scaling as $F_{d} \sim(a v)^{3 / 2}$ and, keeping everything else unchanged, would yield $v \sim a^{\beta}$, with $\beta=0$ and $1 / 5$ for the $3 \mathrm{D}$ and $2 \mathrm{D}$ cases, respectively, a dependence much weaker than that observed experimentally. However, given the expected thickness for the boundary layer, finite-depth effects on swimming velocities should not be observed, in 
contradiction with Fig. 6, suggesting that the boundary layer picture does not capture all the physics at play. What the drag force and the flow structure are in the real system-in the presence of Marangoni effects and with swimmer of finite thickness in particular-remains to be clarified.

(iii) Absence of excluded volume. The model assumes free flow within the swimmer, which is clearly nonphysical for a solid particle. For the phoretic spherical swimmer, improvement of the point-source approach is possible as shown in Sec. A of S.M. [62]. With camphor disks, the free-flow assumption is less drastic: Since the fluid remains mostly below the swimmer, the advection-diffusion of the chemical is less affected by excluded volume.

(iv) Pure 2D/3D case. Assuming that the surfactant exclusively lies either at the interface (insoluble limit) or in the bulk (instantaneous surface-bulk equilibration) is useful to make the model tractable but should be seen as the two limiting cases of a more general phenomenon. In principle, one should consider two fields, the surface and bulk concentrations, which may locally equilibrate, or not, depending on the kinetic of adsorption and desorption. It is interesting to note, though, that in a recent study of steady Marangoni flows induced by a point source [79,83], the case of camphoric acid is better described by the 2D model, suggesting that such a limiting case could be appropriate for our experimental system.

(v) Constant flux. The interaction between a diffusive boundary layer below the swimmer and the growing diffusing layer inside the gel may influence the amount of camphor released. In particular, one could expect higher fluxes at large velocities, implying that big swimmers may swim faster than predicted with the constant flux assumption. Again, a numerical approach is probably required to reach a full understanding.

(vi) Linear equations of state. Following most theoretical treatments, we have chosen in Eq. (16) a linear dependence of surface tension on local concentration. This implies that the concentration remains very far from saturation everywhere in the system, even in the vicinity of the source. Likewise, the possibility of locally saturating the camphor solution has not been considered.

(vii) Punctual release. The camphor release occurs all over the immersed surface of the experimental swimmer, and not only at its center as assumed in the model. However, as shown in Appendix B, this assumption may be inconsequential in the regime considered.

To conclude, the point-source model is sufficient to capture two robust features of camphor disk swimming: a symmetrybreaking propulsion mechanism and a sublinear increase of velocity with swimmer size. On the other hand, reaching a quantitative prediction on the velocity is likely a much more challenging task that would require significant improvements over all approximations listed above.

\section{CONCLUSION}

To describe the spontaneous motion of a solid chemically active particle, we have developed a generic point-source model which captures within a single framework the essential features of self-propulsion mechanism. We found that phoretic and interfacial swimmers operate in very different regimes. With size typically in the micrometric range, phoretic swimmers generally fall below the threshold for spontaneous motion and their velocity is governed by intrinsic asymmetry, however small it may be. In contrast, the symmetry-breaking mechanism is fully operative in interfacial swimmers, whose swimming regime is dominated by advection and makes small intrinsic asymmetry irrelevant. Experiments on camphor disks confirm this picture.

Since our description involves a host of assumptions that are simple, if not crude, a more accurate treatment is called for but it is worth pointing out the complexity involved in the complete problem. The coupling of dissolution, diffusion, advection of camphor, and Marangoni flows, not to mention evaporation, together with the presence of boundary layers around the swimmer, make analytical progress probably very challenging. A numerical approach appears as essential to describe all phenomena more realistically. This is work in progress.

Because it is sufficient to recapitulate some basic physics while remaining elementary, the point-source model may be a useful zero-order approximation and a convenient starting point to investigate a variety of situations. As regards individual propulsion, we have assumed steady state everywhere. Yet how the swimmer sets into motion and the stability of steady swimming remains to be characterized. Distinct phenomena may be expected in the transient regime, since the chemical wake is history dependent and may lead to memory effects in swimming. In particular, it would be interesting to see whether one could reproduce the different modes (continuous, oscillatory, or no motion) evidenced experimentally $[47,49]$. Another point deserving further attention is the behavior of a swimmer next to a wall. Finally, a basic description of interactions between swimmers could open the way to a better understanding of collective effects in interfacial swimmers first reported more than two centuries ago and still under investigation $[6,15]$.

\section{ACKNOWLEDGMENTS}

We are grateful to G. Davis for preliminary work on the experimental system and to S. Michelin for communicating some data on the phoretic swimmer. We also thank F. Raynal, R. Volk, M. Bourgoin, and F. Nadal for fruitful discussions. This work was supported by French research programs ANR16-CE30-0028 and LABEX iMUST (ANR-10-LABX-0064) of Université de Lyon, within the program "Investissements d'Avenir" (ANR-11-IDEX-0007) operated by the French National Research Agency (ANR).

\section{APPENDIX A: CALCULATION DETAILS}

Here we provide some details on the derivation of results presented in Sec. II B. We consider only the 3D phoretic swimmer, the approach being identical for other cases. A small-Pe expansion of Eq. (12) gives

$$
\begin{aligned}
-\chi & +\left(\frac{3}{M}-1+\frac{3 \chi^{2}}{5}\right) \mathrm{Pe} \\
+ & \left(1+\frac{6 \chi}{5}-\frac{2 \chi^{3}}{7}\right) \mathrm{Pe}^{2}+\mathcal{O}\left(\mathrm{Pe}^{3}\right)=0
\end{aligned}
$$


For $\chi=0$, this reduces to $(3 / M-1) \mathrm{Pe}+\mathrm{Pe}^{2}=0$. Therefore $\mathrm{Pe}=0$ for $M<3$ and $\mathrm{Pe}=1-3 / M$ otherwise. For $M$ close to $M_{c}$, the later expression gives Eq. (13). In the large Péclet regime $(\mathrm{Pe} \rightarrow \infty)$, one finds

$$
\frac{\mathrm{Pe}}{M}-\frac{1}{2 \mathrm{Pe}}+\frac{1}{2 \mathrm{Pe}^{2}}+\mathcal{O}\left(\frac{1}{\mathrm{Pe}}\right) e^{-2(1+\chi) \mathrm{Pe}}=0 .
$$

Neglecting the exponential terms, which is reasonable already for $\mathrm{Pe}=10$, we obtain at lowest order $\mathrm{Pe}=\sqrt{M / 2}$. Using one additional term yields the better approximation given by Eq. (15)

\section{APPENDIX B: NONPUNCTUAL RELEASE}

For the camphor disks considered here, the source is not punctual but uniform over the surface of the swimmer. Let us consider a distributed source, possibly in an inhomogeneous way. How are the above results modified? If we consider only advection (infinite Pe) and a 3D interfacial swimmer, we can obtain a precise answer. Assuming that the source maintain radial symmetry and writing the local flux as $J(\mathbf{r})=$ $\frac{\mathcal{J}}{\pi a^{2}} f(r / a)$, then one finds for the velocity

$$
v^{2}=\beta \frac{J \kappa}{C \eta a},
$$

where the numerical prefactor is

$$
\beta=\frac{2}{\pi} \int_{0}^{1} d p \breve{f}(p), \quad \breve{f}(p)=2 \int_{|p|}^{\infty} \frac{f(u) u d u}{\sqrt{u^{2}-p^{2}}},
$$

that is, $\breve{f}$ is the Abel transform of $f$. Combining the two above equations show that $\beta=1$, whatever the function $f$. The velocity is independent of the release profile. Such a result arises in part because we have assumed a linear dependence of $\gamma$ on concentration [see Eq. (16)]. As a consequence, what matters is only the total amount of chemical behind the swimmer, not its spatial distribution. This conclusion would not hold for nonlinear relations $\gamma(\phi)$ such as those considered in Refs. [6,50].
[1] J. Lighthill, Mathematical Biofluiddynamics (SIAM, Philadelphia, PA, 1975).

[2] Though the word swimming may conjure images of mechanical motion, we follow the literature and use swimmer to refer to any particle that undergoes self-propelled motion.

[3] R. Kapral, Perspective: Nanomotors without moving parts that propel themselves in solution, J. Chem. Phys. 138, 020901 (2013).

[4] J. L. Moran and J. D. Posner, Phoretic self-propulsion, Annu. Rev. Fluid Mech. 49, 511 (2017).

[5] S. Nakata, M. Nagayama, H. Kitahata, N. J. Suematsu, and T. Hasegawa, Physicochemical design and analysis of selfpropelled objects that are characteristically sensitive to environments, Phys. Chem. Chem. Phys. 17, 10326 (2015).

[6] H. Nishimori, N. J. Suematsu, and S. Nakata, Collective behavior of camphor floats migrating on the water surface, J. Phys. Soc. Jpn. 86, 101012 (2017).

[7] N. J. Suematsu and S. Nakata, Evolution of self-propelled objects: From the viewpoint of nonlinear science, Chem. Eur. J. 24, 6308 (2018).

[8] J. L. Anderson, Colloid transport by interfacial forces, Annu. Rev. Fluid Mech. 21, 61 (1989).

[9] P. Mitchell, Hypothetical thermokinetic and electrokinetic mechanisms of locomotion in micro-organisms, Proc. R. Phys. Soc. Edinb. 25, 32 (1956).

[10] P. Mitchell, Self-electrophoretic locomotion in microorganisms: Bacterial flagella as giant ionophores, FEBS Lett. 28, 1 (1972).

[11] W. F. Paxton, K. C. Kistler, C. C. Olmeda, A. Sen, S. K. St. Angelo, Y. Cao, T. E. Mallouk, P. E. Lammert, and V. H. Crespi, Catalytic nanomotors: Autonomous movement of striped nanorods, J. Am. Chem. Soc. 126, 13424 (2004).

[12] J. R. Howse, R. A. L. Jones, A. J. Ryan, T. Gough, R. Vafabakhsh, and R. Golestanian, Self-Motile Colloidal Particles: From Directed Propulsion to Random Walk, Phys. Rev. Lett. 99, 048102 (2007).
[13] H.-R. Jiang, N. Yoshinaga, and M. Sano, Active Motion of a Janus Particle by Self-thermophoresis in a Defocused Laser Beam, Phys. Rev. Lett. 105, 268302 (2010).

[14] I. Buttinoni, G. Volpe, F. Kümmel, G. Volpe, and C. Bechinger, Active Brownian motion tunable by light, J. Phys.: Condens. Matter 24, 284129 (2012).

[15] Romieu, Mémoire sur une nouvelle végétation chimique faite avec le camphre, Mem. Acad. R. Sci. 443 (1756).

[16] Dutrochet, Recherches sur la cause des mouvements que présente le camphre placé à la surface de l'eau, et sur la cause de la circulation chez le Chara, C. R. Acad. Sci. (1841).

[17] N. Joly and A. Boisgiraud, Nouvelles recherches sur les mouvements du camphre et de quelques autres corps placés à la surface de l'eau et du mercure (Rovet, Paris 1842).

[18] H. Devaux, Mouvements spontanés de certains corps à la surface de quelques liquides, La nature 777, 331 (1888).

[19] W. H. Larrabee, The surface tension of liquids, Pop. Sci. Month. 35, 591 (1889).

[20] H. Jin, A. Marmur, O. Ikkala, and R. H. A. Ras, Vapour-driven Marangoni propulsion: Continuous, prolonged and tunable motion, Chem. Sci. 3, 2526 (2012).

[21] L. J. Burton, N. Cheng, and J. W. M. Bush, The cocktail boat, Integr. Comp. Biol. 54, 969 (2014).

[22] D. L. Hu, The Hydrodynamics of Water-walking Insects and Spiders, Ph.D. thesis, MIT, 2001.

[23] J. W. M. Bush and D. L. Hu, Walking on Water: Biolocomotion at the interface, Annu. Rev. Fluid Mech. 38, 339 (2006).

[24] C. Bechinger, R. Di Leonardo, H. Löwen, C. Reichhardt, G. Volpe, and G. Volpe, Active Brownian particles in complex and crowded environments, Rev. Mod. Phys. 88, 045006 (2016).

[25] E. Heisler, N. J. Suematsu, A. Awazu, and H. Nishimori, Swarming of self-propelled camphor boats, Phys. Rev. E 85, 055201(R) (2012).

[26] F. Ginot, I. Theurkauff, F. Detcheverry, C. Ybert, and C. CottinBizonne, Aggregation-fragmentation and individual dynamics of active clusters, Nat. Commun. 9, 696 (2018). 
[27] J. E. Satterwhite-Warden, D. K. Kondepudi, J. A. Dixon, and J. F. Rusling, Co-operative motion of multiple benzoquinone disks at the air-water interface, Phys. Chem. Chem. Phys. 17, 29891 (2015).

[28] Y. S. Ikura, E. Heisler, A. Awazu, H. Nishimori, and S. Nakata, Collective motion of symmetric camphor papers in an annular water channel, Phys. Rev. E 88, 012911 (2013).

[29] S. Soh, M. Branicki, and B. A. Grzybowski, Swarming in shallow waters, J. Phys. Chem. Lett. 2, 770 (2011).

[30] B. A. Grzybowski, C. E. Wilmer, J. Kim, K. P. Browne, and K. J. M. Bishop, Self-assembly: From crystals to cells, Soft Matter 5, 1110 (2009).

[31] R. Golestanian, T. B. Liverpool, and A. Ajdari, Designing phoretic micro- and nano-swimmers, New J. Phys. 9, 126 (2007).

[32] S. Michelin and E. Lauga, Phoretic self-propulsion at finite Péclet numbers, J. Fluid Mech. 747, 572 (2014).

[33] E. Yariv and S. Michelin, Phoretic self-propulsion at large Péclet numbers, J. Fluid Mech. 768, R1 (2015).

[34] D. Sondak, C. Hawley, S. Heng, R. Vinsonhaler, E. Lauga, and J.-L. Thiffeault, Can phoretic particles swim in two dimensions? Phys. Rev. E 94, 062606 (2016).

[35] S. Michelin, E. Lauga, and D. Bartolo, Spontaneous autophoretic motion of isotropic particles, Phys. Fluids 25, 061701 (2013).

[36] P. de Buyl, A. S. Mikhailov, and R. Kapral, Self-propulsion through symmetry breaking, Europhys. Lett. 103, 60009 (2013).

[37] D. Yamamoto, A. Mukai, N. Okita, K. Yoshikawa, and A. Shioi, Catalytic micromotor generating self-propelled regular motion through random fluctuation, J. Chem. Phys. 139, 034705 (2013).

[38] D. A. Gregory and S. J. Ebbens, Symmetrical catalytically active colloids collectively induce convective flow, Langmuir 34, 4307 (2018).

[39] Given their sub-micrometric size, it is probably difficult to ensure that the particles uniformly covered with Pt used in Ref. [37] are perfectly spherical. As regards Ref. [38], the appearance of convective flows in suspension of isotropic particles appears to be a collective effect, as it occurs only above a threshold volume fraction.

[40] Z. Izri, M. N. van der Linden, S. Michelin, and O. Dauchot, Self-Propulsion of Pure Water Droplets by Spontaneous Marangoni-Stress-Driven Motion, Phys. Rev. Lett. 113, 248302 (2014).

[41] M. Schmitt and H. Stark, Swimming active droplet: A theoretical analysis, Europhys. Lett. 101, 44008 (2013).

[42] M. Schmitt and H. Stark, Marangoni flow at droplet interfaces: Three-dimensional solution and applications, Phys. Fluids 28, 012106 (2016).

[43] M. Morozov and S. Michelin, Self-propulsion near the onset of Marangoni instability of deformable active droplets, J. Fluid Mech. 860, 711 (2019).

[44] S. Nakata, J. Kirisaka, Y. Arima, and T. Ishii, Self-motion of a camphanic acid disk on water with different types of Surfactants, J. Phys. Chem. B 110, 21131 (2006).

[45] N. J. Suematsu, Y. Ikura, M. Nagayama, H. Kitahata, N. Kawagishi, M. Murakami, and S. Nakata, Mode-switching of the self-motion of a camphor boat depending on the diffusion distance of camphor molecules, J. Phys. Chem. C 114, 9876 (2010).

[46] K. Iida, N. J. Suematsu, Y. Miyahara, and H. Kitahata, Experimental and theoretical studies on the self-motion of a phenanthroline disk coupled with complex formation, Phys. Chem. Chem. Phys. 12, 1557 (2010).

[47] S. Nakata, Y. Matsuda, Y. S. Ikura, A. Takeda, and S. Izumi, Mode change in the self-motion of a benzoquinone disk coupled with a NADPH system, ChemPhysChem 13, 520 (2012).

[48] Y. Matsuda, N. J. Suematsu, H. Kitahata, Y. S. Ikura, and S. Nakata, Acceleration or deceleration of self-motion by the Marangoni effect, Chem. Phys. Lett. 654, 92 (2016).

[49] V. S. Akella, D. K. Singh, S. Mandre, and M. M. Bandi, Dynamics of a camphoric acid boat at the air-water interface, Phys. Lett. A 382, 1176 (2018).

[50] M. Nagayama, S. Nakata, Y. Doi, and Y. Hayashima, A theoretical and experimental study on the unidirectional motion of a camphor disk, Physica D 194, 151 (2004).

[51] Y. Koyano, T. Sakurai, and H. Kitahata, Oscillatory motion of a camphor grain in a one-dimensional finite region, Phys. Rev. E 94, 042215 (2016).

[52] Y. Koyano, N. J. Suematsu, and H. Kitahata, Rotational motion of a camphor disk in a circular region, Phys. Rev. E 99, 022211 (2019).

[53] A. Mikhailov and D. Meinköhn, Self-motion in physicochemical systems far from thermal equilibrium, Lect. Notes Phys. 484, 334 (2007).

[54] E. Lauga and A. M. J. Davis, Viscous Marangoni propulsion, J. Fluid Mech. 705, 120 (2012).

[55] A. Mikhailov and V. Calhenbur, From Cells to Societies (Springer, Berlin, 2002).

[56] N. J. Suematsu, T. Sasaki, S. Nakata, and H. Kitahata, Quantitative estimation of the parameters for self-motion driven by difference in surface tension, Langmuir 30, 8101 (2014).

[57] The case of self-thermophoresis might be treated along similar lines.

[58] Since the phoretic velocity is $\mathcal{U} \sim \mathcal{M} \phi / a$ from Eq. (7) and the local diffusion flux is $J=\mathcal{J} / a^{2} \sim D \nabla \phi \sim D \phi / a$, one gets for the corresponding Péclet number $\mathcal{U} a / D=M$.

[59] The surface tension is assumed to depend on the chemical concentration in the immediate vicinity of the surface.

[60] Since the typical velocity is $\mathcal{U} \sim \kappa \phi / C \eta$ from Eq. (17) and the diffusion flux is $\mathcal{J} / a^{2} \sim D \phi / a$ as in the phoretic case, one gets $\mathcal{U} a / D=M$.

[61] E. Yariv, Two-dimensional phoretic swimmers: The singular weak-advection limits, J. Fluid Mech. 816, R3 (2017).

[62] See Supplemental Material at http://link.aps.org/supplemental/ 10.1103/PhysRevE.99.062605 on four items: (A) "Point+ dipole" model for 3D phoretic swimmer. (B) Influence of pool solution on swimming velocity of camphor disks. (C) Measurement of $\mathcal{J}(t)$ and $C_{\mathrm{sol}}$. (D) Boundary effect and exclusion zone.

[63] The amount $\mathcal{J} d t$ released during a time interval $d t$ is found in a box of length $v d t$ and lateral dimension(s) $w$.

[64] For instance, taking Eq. (3) of Ref. [12] in the limit of very large peroxide concentration gives a maximum rate $J \simeq$ $\mathcal{N}_{A} / 10 \mathrm{~m}^{-2} \mathrm{~s}^{-1}$.

[65] $l_{\text {ads }}$ is thus the thickness of liquid such that the bulk and surface amounts of chemical are the same. The derivation of Eq. (29) 
involves several approximations. First, we consider the simplest equation of state $\gamma=\gamma_{o}-k_{B} T \Gamma$, with $\Gamma$ the surface concentration. Second, we assume that equilibrium is instantaneously established between the bulk and the surface, and that the latter remains far from saturation, so that the bulk concentration $c$ and the surface concentration are linearly related $\Gamma=l_{\text {ads }} c$. The $\kappa$ parameter of Eq. (16) is therefore $\kappa=l_{\text {ads }} k_{B} T$.

[66] See the experimental measurement for camphor in the Supplemental Material of Ref. [56].

[67] S. Soh, K. J. M. Bishop, and B. A. Grzybowski, Dynamic selfassembly in ensembles of camphor boats, J. Phys. Chem. B 112, 10848 (2008).

[68] The Merck Index: An Encyclopedia of Chemicals, Drugs, and Biologicals, edited by M. J. O’Neil (RSC Publishing, Cambridge, 2013).

[69] Canon PowerShot S110 camera, taking each second 30 frames of $1280 \times 720$ pixels or 24 frames of $1920 \times 1080$ pixels.

[70] J. C. Crocker and D. G. Grier, Methods of digital video microscopy for colloidal studies, J. Colloid Interface Sci. 179, 298 (1996).

[71] H. K. Yuen, J. Princen, J. Illingworth, and J. Kittler, Comparative study of Hough Transform methods for circle finding, Image Vis. Comput. 8, 71 (1990).

[72] H. Kitahata, H. Yamamoto, M. Hata, Y. S. Ikura, and S. Nakata, Relaxation dynamics of the Marangoni convection roll structure induced by camphor concentration gradient, Colloids Surf. A 520, 436 (2017).

[73] J. Happel and H. Brenner, Low Reynolds Number Hydrodynamics (Kluwer, Amsterdam, 1983).
[74] J. M. P. Q. Delgado, Molecular diffusion coefficients of organic compounds in water at different temperatures, J. Phase Equilib. Diffus. 28, 427 (2007).

[75] The glass pool has a circular shape with radius $a=11.5 \mathrm{~cm}$ and depth $h=10 \mathrm{~cm}$. To avoid swimming along the wall, the boundary is equipped with a floating plastic edge whose flowerlike shape favors reinjection into the center of the pool.

[76] The velocity is seen to increase with $d$ when the disk starts to protrude outside of the boat.

[77] Note the difference with the assumption of Table I. When using the $2 \mathrm{D}$ model, we simply assume that all camphor released eventually lies at the interface.

[78] For the range of asymmetry considered here $\left(\chi^{\prime}<0.06\right)$, the prefactor $\left(1-\chi^{\prime}\right)^{-1 / 3} \simeq 1+\chi^{\prime} / 3+\mathcal{O}\left(\chi^{\prime 2}\right)$ amounts to a fewpercentages correction.

[79] S. Mandre, Axisymmetric spreading of a surfactant driven by self-imposed Marangoni stress under simplified transport, J. Fluid Mech. 832, 777 (2017).

[80] H. Kitahata and N. Yoshinaga, Effective diffusion coefficient including the Marangoni effect, J. Chem. Phys. 148, 134906 (2018).

[81] T. Bickel, Spreading dynamics of reactive surfactants driven by Marangoni convection, Soft Matter 15, 3644 (2019).

[82] H. Ockendon and J. Ockendon, Viscous Flow (Cambridge University Press, Cambridge, 1995).

[83] M. Bandi, V. Akella, D. Singh, R. Singh, and S. Mandre, Hydrodynamic Signatures of Stationary Marangoni-Driven Surfactant Transport, Phys. Rev. Lett. 119, 264501 (2017). 